Title : will be set by the publisher

Editors : will be set by the publisher

EAS Publications Series, Vol. ?, 2018

\title{
SOLITON SOLUTIONS IN RELATIVISTIC FIELD THEORIES AND GRAVITATION
}

\author{
Joaquin Diaz-Alonso $^{1,2}$ and Diego Rubiera-Garcia ${ }^{2}$
}

\begin{abstract}
We report on some recent results on a class of relativistic lagrangian field theories supporting non-topological soliton solutions and their applications in the contexts of Gravitation and Cosmology. We analyze one and many-components scalar fields and gauge fields.
\end{abstract}

\section{Introduction}

The well-known Born-Infeld (BI) model (Born \& Infeld 1934) was historically proposed to remove the divergence of the electron's self-energy in classical electrodynamics. Aside from electrostatic soliton solutions in three space dimensions, the ' model exhibits dyon and bidyon-like solutions (Chernitskii[1999), electric-magnetic duality (Gibbons \& Rasheed 1995) and special properties of wave propagation, be' longing to the class of "completely exceptional" theories (Boillat 1970).

Recently there has been a renewed interest in BI theory and its non-abelian ' extensions since they appear at different levels of string theory (Gibbons 1998). However, there are many other physical contexts where BI-like models have been ' used, such as the description of dark energy in Cosmology (e.g. Füzfa \& Alimi 2006) and the phenomenological description of the nucleon structure (Deser et al 1976. Pavlovski 2002).

On the other hand, many studies on generalized electromagnetic and gauge field theories coupled with gravitation have been devoted to the analysis of particle-like solutions. Although several theorems of the 70's (Coleman 1977) forbid the existence of static, finite-energy solutions of the pure Yang-Mills theory, such solutions were found in the Einstein-Yang-Mills system (Bartnick \& McKinnon 1988). Later it was shown that similar glueball solutions also exist in BI-like models in flat space (Gal'tsov \& Kerner 2000) as well as in curved space (e.g. Wirschins et al 2001) (see Volkov \& Gal'tsov (1999) for a review and references on non-abelian solitons).

\footnotetext{
1 LUTH, Observatoire de Paris, CNRS, Université Paris Diderot. 5 Place Jules Janssen, 92190 Meudon, France

2 Departamento de Fisica, Universidad de Oviedo. Avda. Calvo Sotelo 18, E-33007 Oviedo, Asturias, Spain
} 
Title : will be set by the publisher

\section{The models}

The BI generalization of classical electrodynamics is by no means unique and, from the point of view of soliton solutions, BI theory is only a particular example of a large class, which has been exhaustively determined in Diaz-Alonso \& Rubiera-Garcia (2007). Here we deal with the generalized gauge field problem, by considering gauge-invariant lagrangians which are given functions $L=\varphi(X, Y)$ of the two quadratic field invariants $X=-\frac{1}{2} \sum_{a} F_{\mu \nu}^{a} F^{\mu \nu a} ; Y=-\frac{1}{2} \sum_{a} F_{\mu \mu}^{a} F^{* \mu \nu a}$, defined in a domain $\left(\Omega \subseteq \Re^{2}\right.$ ) which is assumed to be open and connected and including the vacuum $(X=Y=0)$. In calculating the field invariants we use the ordinary definition of the trace, although other definitions are possible (Tseytlin 1997). We also require the condition $\varphi(X, Y)=\varphi(X,-Y)$ to be satisfied, in order to preserve parity invariance. Moreover we restrict our analysis to "physically admissible theories", which we define by the requirement of the vanishing of the vacuum energy $(\varphi(0,0)=0)$, as well as the positive definiteness of the energy, which demands the minimal necessary and sufficient condition

$$
\rho^{s} \geq\left(X+\sqrt{X^{2}+Y^{2}}\right) \frac{\partial \varphi}{\partial X}+Y \frac{\partial \varphi}{\partial Y}-\varphi(X, Y) \geq 0
$$

to be satisfied in the entire domain of definition $(\Omega)$. The field equations read

$$
\sum_{b} D_{a b \mu}\left[\frac{\partial \varphi}{\partial X} F^{\mu \nu b}+\frac{\partial \varphi}{\partial Y} F^{* \mu \nu b}\right]=0
$$

where $D_{a b \mu} \equiv \delta_{a b} \partial_{\mu}+g \sum_{c} C_{a b c} A_{c \mu}$. We next consider the electrostatic spherically symmetric solutions $(\mathrm{ESS})\left(\vec{E}_{a}(r)=-\vec{\nabla}\left(\phi_{a}(r)\right)=-\phi_{a}^{\prime}(r) \frac{\vec{r}}{r} ; \vec{H}_{a}=0\right)$. When this substitution is done in (2.2) we get two sets of equations

$$
\nu=0 \rightarrow \vec{\nabla}\left(\frac{\partial \varphi}{\partial X} \vec{\nabla} \phi_{a}(r)\right)=0 ; \nu=i=1,2,3 \rightarrow \sum_{b c} C_{a b c} \phi_{b}(r) \phi_{c}^{\prime}(r)=0
$$

The first set leads to the first integrals $r^{2} \frac{\partial \varphi}{\partial X} \phi^{\prime}(r)=Q_{a}\left(Q_{a}\right.$ being integration constants, identified as color charges) which coincide with the set of first integrals of the field equations for static, spherically symmetric solutions (SSS) of a multicomponent scalar field theory with a lagrangian density given by

$$
L=f\left(\sum_{a} \partial_{\mu} \phi_{a} \partial^{\mu} \phi_{a}\right) \equiv f(X)=-\varphi(-X, Y=0)
$$

The solutions of Eqs.(2.3) for the multiscalar or gauge cases take the form $\phi_{a}(r)=$ $\frac{Q_{a}}{Q}\left(\phi(r, Q)+\alpha_{a}\right)$, where $Q=\sqrt{Q_{a}^{2}}$ is the mean-square (scalar or color) charge and $\alpha_{a}$ are integration constants. The function $\phi(r, Q)$ is the solution of the one-component scalar field theory resulting from the restricted lagrangian $f(X=$ $\left.\partial_{\mu} \phi \partial^{\mu} \phi\right)$. In the gauge case the second set of equations (2.3) restricts the possible 


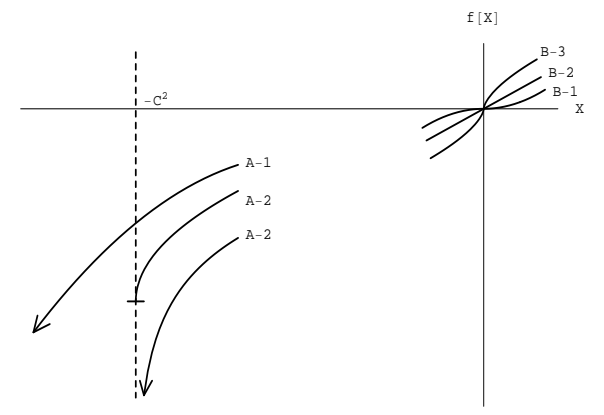

Fig. 1. Different possible central and asymptotic behaviors of the admissible models

values of the integration constants $\alpha_{a}=\frac{Q_{a}}{Q} \alpha$ and then the ESS solutions take the form $\phi_{a}(r)=\frac{Q_{a}}{Q}(\phi(r, Q)+\alpha)$ where now $Q$ is the mean-square color charge.

Thus, the form of the ESS solutions of generalized gauge field theories coincides with that of the SSS solutions of an associated one-component scalar field theory. The energy of the ESS solutions, when finite, is twofold the energy of the associated SSS solutions, which reads

$$
\varepsilon(Q)=-4 \pi \int_{0}^{\infty} r^{2} f\left(-\phi^{\prime 2}(r)\right) d r=Q^{3 / 2} \varepsilon(Q=1) .
$$

The requirement of convergence of this integral leads to a classification of the

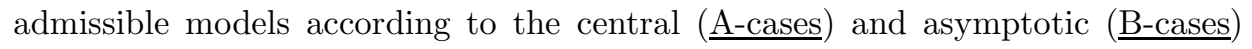
behaviors of the soliton field strength (see Fig.1). Cases $A-1$ and $A$-2 correspond to infinite (but integrable) and finite soliton field strengths, respectively. Cases $B-1$, $B-2$ and $B-3$ correspond to asymptotic dampings of the soliton field strength which are slower than coulombian, coulombian, or faster than coulombian, respectively (Diaz-Alonso \& Rubiera-Garcia 2007).

For each admissible scalar model supporting finite-energy SSS solutions there exists an infinite family of admissible gauge field theories supporting similar finiteenergy ESS solutions, obtained from (2.4) and the admissibility conditions (2.1).

The linear stability of the SSS and ESS solutions requires the energy (2.5) to be a minimum against small charge-preserving perturbations of the fields and potentials in the gauge and scalar cases. In the latter we found that all finiteenergy SSS solutions of admissible models are always linearly stable while in the former the following supplementary condition for stability must be satisfied

$$
\frac{\partial \varphi}{\partial X}-2 X \frac{\partial^{2} \varphi}{\partial Y^{2}}>0, \forall(X, Y=0)
$$

As a non-trivial example of this class of soliton-supporting theories we introduce here the family of generalized electromagnetic field models

$$
\varphi(X, Y)=X / 2+\lambda X^{a}+\beta Y^{2}(a>3 / 2, \beta>0),
$$


Title : will be set by the publisher

which is the simplest generalization of a family of scalar models (defined from (2.7) by setting $\beta=0$ ) supporting SSS soliton solutions, which has been analyzed in Diaz-Alonso \& Rubiera-Garcia (2007). This generalized electromagnetic family fulfills the admissibility conditions (2.1) and supports ESS solitons. For certain values of the parameters in the electromagnetic case $(\lambda<0, a=2)$ the non-linear term has the form of the decoupled part of the four-vertex contribution to the effective lagrangian of quantum electrodynamics.

\section{Conclusions and outlook}

In summary, we have analyzed scalar and generalized gauge field theories supporting soliton solutions. Aside from BI-like models which have been widely used during the last few years, other models have also recently attracted attention in search of self-gravitating scalar, electromagnetic and gauge field solitons as well as regular charged solutions (Ayon-Beato \& Garcia 1999). The class of models considered here can also be useful in other physical contexts. For instance, in the extension of the description of dark energy in Cosmology, as a non-canonical scalar field (Armendariz-Picon \& Lim 2005) or as a gauge field governed by generalized

actions (Dyadichev et al 2002), in the phenomenological description of nucleon structure, in generalized gauge theories in higher dimensions, glueballs, etc.

\section{References}

Armendariz-Picon, C. \& Lim, E.A. 2005, JCAP, 0508, 007.

Ayon-Beato, E. \& Garcia, A. 1999, Gen. Rel. Grav., 31, 629.

Bartnick, R. \& McKinnon, J. 1988, Phys. Rev. Lett., 61, 141.

Boillat, G. 1970, J. Math. Phys., 11, 941.

Born, M. \& Infeld, L. 1934, Proc. R. Soc. London. A, 144, 425.

Chernitskii, A.A. 1999, J. High. Ener. Phys., 9912, 010.

Coleman, S. 1977, Comm. Math. Phys., 55, 113.

Deser, S., Duff, M.J., \& Isham, C.J. 1976, Nucl. Phys. B, 114, 29

Diaz-Alonso, J. \& Rubiera-Garcia, D. 2007, Phys. Lett. B, 653, 445.

Diaz-Alonso, J. \& Rubiera-Garcia, D. 2007, Phys. Lett. B, 657, 257.

Diaz-Alonso, J. \& Rubiera-Garcia, D. "A study on relativistic lagrangian field theories with non-topological soliton solutions", Preprint (2007). To be published.

Dyadichev, V., Gal'tsov, D., Zorin, A., \& Zotov, M. 2002, Phys. Rev. D, 65, 084007.

Füzfa, A. \& Alimi, J.-M. 2006, Phys. Rev. D, 73, 023520.

Gal'tsov, D. \& Kerner, R. 2000, Phys. Rev. Lett., 84, 5955.

Gibbons, G.W. \& Rasheed, D.A. 1995, Nucl. Phys. B, 454, 185.

Gibbons, G.W. 1998, Nucl. Phys. B, 514, 603.

Pavlovskii, O.V. 2002, Phys. Lett. B, 538, 202.

Tseytlin, A. 1997, Nucl. Phys. B, 501, 41.

Volkov, M.S. \& Gal'tsov, D.V. 1999, Physics Reports, 319, 1.

Wirschins, M., Sood, A., \& Kunz, J. 2001, Phys. Rev. D, 63, 084002. 